\title{
Exponential Galaxy Disks from Stellar Scattering
}

\author{
Bruce G. Elmegreen \\ IBM Research Division, T.J. Watson Research Center, 1101 Kitchawan Road, Yorktown \\ Heights, NY10598,bge@watson.ibm.com \\ and \\ Curtis Struck \\ Department of Physics and Astronomy, Iowa State University, Ames, IA 50011, \\ curt@iastate.edu
}

\begin{abstract}
Stellar scattering off of orbiting or transient clumps is shown to lead to the formation of exponential profiles in both surface density and velocity dispersion in a two-dimensional non-self gravitating stellar disk with a fixed halo potential. The exponential forms for both nearly-flat rotation curves and near-solid body rotation curves. The exponential does not depend on initial conditions, spiral arms, bars, viscosity, star formation, or strong shear. After a rapid initial development, the exponential saturates to an approximately fixed scale length. The inner exponential in a two-component profile has a break radius comparable to the initial disk radius; the outer exponential is primarily scattered stars.
\end{abstract}

Subject headings: galaxies: evolution — galaxies: formation — galaxies: structure

\section{Introduction}

Spiral galaxies have exponential radial profiles (Freeman 1970; van der Kruit 2002) with a nearly uniform central surface brightness for early and intermediate Hubble types and a trend toward lower central brightnesses for later types (de Jong 1996; Graham \& de Blok 2001). The scale length is also independent of Hubble type for early and intermediate types (de Jong 1996), but increases with increasing galaxy mass (Fathi 2010) within those types. Generally galaxies with larger scale lengths have fainter central brightnesses (Fathi 2010).

The radial profiles themselves show distinct types, originally classified by Freeman (1970) and studied more recently by Pohlen \& Trujillo (2006), Erwin et al. (2008), Herrmann et al. 
(2013) and others. Type I is a uniform exponential with a constant scale length, Type II is a double exponential with a steeper outer part, and Type III is a double exponential with a shallower outer part. These types correlate with Hubble type in the sense that Type IIs are more common in late type galaxies (Pohlen \& Trujillo 2006; Gutiérrez et al. 2011; Herrmann et al. 2013). Late types also have a smaller ratio of the break radius to the inner scale length (Pohlen \& Trujillo 2006; Hunter \& Elmegreen 2006).

The are several theories for the origin of the exponential shape. A uniform-density, uniformly rotating spherical halo that collapses to a disk with no angular momentum redistribution between mass elements (Mestel 1963) has a distribution of mass as a function of angular momentum that is nearly the same as that of a self-gravitating exponential profile out to a radius $R \sim 6 R_{\mathrm{D}}$ scale lengths (Freeman 1970). Early observations of outer disk angular momentum distributions supported such idealized collapse (Crampin \& Hoyle 1964) as did early models of disk formation (Fall \& Efstathiou 1980). Efstathiou (2000) showed, however, that no exponential formed this way can go out very far if it comes from a power law halo because the outer disk mass is exponentially small and has to come from a very narrow range of halo radii where the range of angular momenta is too narrow compared to that required for the disk (Ferguson \& Clarke 2001). Now we know that some galaxies have exponential disks out to $\sim 10 R_{\mathrm{D}}$ (e.g., Weiner et al. 2001; Bland-Hawthorn et al. 2005).

The Mestel (1963) model was popular when exponential disks seemed to have abrupt outer truncations at $\sim 4.5 R_{\mathrm{D}}$ (e.g., Dalcanton et al. 1997), in which case this outer radius could be related to the maximum angular momentum of the initial halo gas. Observations now show a transition to a second exponential or other peripheral stellar structure at about this radius with no sharp truncation even in extremely deep surveys based on photometry (e.g., Hunter et al. 2011) or star counts (Saha et al. 2010; McConnachie et al. 2010; Vlajić et al. 2011; Grossi et al. 2011; Radburn-Smith et al. 2012; Barker et al. 2012). The assumption of specific angular momentum conservation is also not made anymore because tidal torques, spiral arms, bars, mergers and continuing gas accretion redistribute the disk angular momentum.

A second model proposes that the exponential shape forms through secular processes when the viscous accretion rate in the disk is proportional to the star formation rate Lin \& Pringle 1987; Yoshii \& Sommer-Larsen 1989; Ferguson \& Clarke 2001; Wang et al. 2009). This model requires shear and works for spiral galaxies, but not for dwarf Irregulars that have exponential or double-exponential disks like spirals, and sometimes out to $\sim 8 R_{\mathrm{D}}$ as well (Hunter et al. 2011), but nearly solid body rotation in at least the inner parts. Hunter \& Elmegreen (2006) also pointed out that there is no obvious relation between the break radius in a dwarf Irregular and the turn-over radius of the rotation curve. 
A third model involves a galaxy bar, which redistributes disk angular momentum and can make an exponential from some initially different distribution (Hohl 1971). Bars also make double exponentials with the outer part just beyond the outer Lindblad resonance of the spirals that the bar generates (Debattista et al. 2006; Foyle et al. 2008). Bars are not present in all disks, however, nor are there bulge-like remnants of bars in late type galaxies even though late types dominate those with double exponentials (Pohlen \& Trujillo 2006). Neither is there a correlation between the presence of bars and exponentials or double exponentials in dwarf Irregular galaxies (Hunter \& Elmegreen 2006). High redshift galaxies, out to $z=5.8$ in Fathi et al. (2012), have exponential disks as well, so this shape has to arise quickly.

Additional models consider possible origins for the break in a single exponential, making it double, such as a threshold in star formation at the break radius (Fall \& Efstathiou 1980; Foyle et al. 2008), which either terminates the disk at that point or makes an outer exponential that falls more steeply than the inner (Elmegreen \& Hunter 2006). Grazing encounters with other galaxies can make a double exponential with the outer one more shallow (Younger et al. 2007; Peñarrubia et al. 2006). Models with firm star formation thresholds require a mechanism to get stars beyond the threshold, and a good candidate for such a mechanism is star scattering by spiral arms (Sellwood \& Binney 2002; Roškar et al. 2008; Martínez-Serrano et al. 2009). Spiral scattering with a star formation threshold makes the outer disk come from the inner disk, and it predicts a color profile that gets bluer with increasing radius in the star formation part and then redder with radius outside of that in the scattered part. Such color profiles are observed in both local (Bakos et al. 2008) and redshift $\sim 1$ galaxies (Azzollini et al. 2008). However, two-component surface brightness profiles with such a color distribution convert into single-exponential mass profiles that seem too regular to be explained by a two-component (star formation plus scattering) model (Bakos et al. 2008). Also dwarf Irregular galaxies with double exponentials have no spiral arms to do the scattering. There are problems with the encounter theory as well: Gutiérrez et al. (2011) point out that Type III's are not just Type I's with their outer parts spread out because the III's are brighter inside.

Numerical simulations of galaxy formation get exponential and double-exponential disks under realistic cosmological conditions, so we might ask how they do it. An essential ingredient for a realistic disk is star formation feedback (Robertson et al. 2004), but the primary role of this feedback is to prevent the gas disk from getting too small. The actual exponential shape that results in these and other simulations is not specifically questioned. Simulations with double exponentials produce them from initial single exponentials, but again the exponential shape itself is not explained. Perhaps the exponential results from a combination of processes listed above, even though no single mechanism can be applied to all galaxies. Or, 
perhaps there is an additional ingredient in these simulations and in real galaxies that has not been specifically noted.

The most stringent test for exponential models seems to be dwarf Irregular galaxies, as noted above. They have little shear, no spirals, are often non-barred, and yet have an underlying disk plus star formation that follows an exponential distribution out to 6 or more scale lengths (to $30 \mathrm{mag} / \operatorname{arcsec}^{2}$ in V-band; Hunter et al. 2011) - long into the regime where the disk seems to be gravitationally stable (van Zee et al. 1997; Hunter et al. 2011). They are also harassed by encounters with galaxies in their groups or by smaller companions (Pustilnik et al. 2001), and many could be subject to continuing gas accretion (van Zee et al. 1998; Wilcots \& Miller 1998). Star formation sometimes mimics the underlying exponential so closely (also in spirals; Shi et al. 2011; Hunter et al. 2013) that it is unclear whether the total stellar exponential is made from the accumulated history of star formation at each radius, or if star formation follows the stars which take an exponential form for other reasons. As discussed in Hunter et al. (2013), the first case seems unlikely because then the dense molecular gas that forms stars has to know about the gas distribution in the whole disk; the second case is more reasonable as it requires the stars to continuously migrate to smooth out non-exponential perturbations. The problem is that in dwarf Irregulars, the stars have to do this migration without spirals, bars or significant shear.

Here we run simple experiments of passive stellar scattering in rotating thin disks that are subject to steady or transient perturbations from point-like gravitating objects. Such objects could be giant star-forming regions in the case of dwarf Irregulars (Hunter et al. 2011) or they could be clumps in high-redshift galaxies (Bournaud et al. 2007). They could also be dense halo objects like dark-matter minihalos that pass through the disk. We find that perturbations like this can make an exponential surface density distribution from an initially flat distribution, and that the distribution of stellar velocity dispersion also becomes exponential from an initially uniform value, with a scale length somewhat longer than for the mass. This result for the dispersion is interesting because it makes the scale height more constant than it would be with the surface density decreasing alone. A near-uniform disk scale height is another observational constraint on the origin of exponential disks (e.g., de Grijs \& Peletier 1997).

\section{Models}

The simplest model for disk evolution with stellar scattering consists of two-dimensional orbital integrations of non-interacting test particles (stars) in centrifugal balance in a fixed potential (halo), along with softened, point-mass gravitating particles (clumps) that are 
also in the disk. Time integrations are carried out with the MATLAB Runge-Kutta routine "ode23." The lack of self-gravity in the stars means that resolution effects are not important. We consider two cases, one with a nearly flat rotation curve and another with a nearly solidbody rotation curve.

\subsection{Nearly-flat rotation curve}

For the first case, the gravitational acceleration of the fixed halo is of the form

$$
g_{\mathrm{F}}(R)=-\frac{G M_{\mathrm{H}}}{H^{2}}\left(\frac{R}{H}\right)^{-1.1}
$$

where $H$ is the halo scale length, and $M_{\mathrm{H}}$ is the halo mass within $H$. This acceleration gives a rotation curve that is slightly falling, $V(R) \propto R^{-0.05}$. The clumps in this model have a softening length $0.01 H$ and are on fixed circular orbits without responding to the stars. The initial disk surface density is independent of radius and has a sharp outer edge at $R=4$ units, as do the clumps. The sharp edge in the stellar distribution does not affect the dynamics because the stars are not self-gravitating.

We take dimensionless length and time units, $H=1$ and $T=H / V_{\text {circ }}=1$ where $V_{\mathrm{H}}^{2}=G M_{\mathrm{H}} / H$. As an example of a large spiral galaxy like the Milky Way, we consider $H=2 \mathrm{kpc}$ and $V_{\mathrm{H}}=220 \mathrm{~km} \mathrm{~s}^{-1}$. Then $T=8.9 \mathrm{Myr}, M_{\mathrm{H}}=H V_{\mathrm{H}}^{2} / G=2.2 \times 10^{10} M_{\odot}$, and the rotation period at $R=H$ is $2 \pi$ code units or $56 \mathrm{Myr}$. At $R=10 \mathrm{kpc}$, the rotation period is $303 \mathrm{Myr}$.

The left-hand side of Figure 1 shows the results of a simulation with 15925 noninteracting stars in initially circular orbits with zero velocity dispersion and with 70 gravitating clumps of mass $0.002 M_{\mathrm{H}}$ each in fixed circular orbits. The figure shows the distribution of these stars (top panel), the radial dependence of stellar surface density (middle panel), and the radial dependence of the rms stellar velocity dispersion (bottom panel) after 300 time units (corresponding to $2.7 \mathrm{Gyr}$ ). The rms velocity dispersion is measured as $\sigma_{\mathrm{rms}}=\left(\sigma_{\mathrm{r}}^{2}+\sigma_{\theta}^{2}\right)^{1 / 2} / 2^{1 / 2}$ for radial and angular components $\sigma_{\mathrm{r}}$ and $\sigma_{\theta}$; it is normalized to $V_{\mathrm{H}}$.

The disk has evolved to have exponential profiles in surface density and velocity dispersion. Without perturber clumps (Fig. 2, black curve), there is no significant evolution of the disk, which keeps its initially flat surface density profile out to the sharp edge. Plots (not shown) of individual stellar trajectories indicate that the co-rotating clumps are scattering stars as they shear by. This scattering drives the overall evolution of the disk toward an exponential profile. The profile is actually double-exponential with the inner part comparable in size to the initial disk. 
The exponential decline of the velocity dispersion in Figure 1 suggests an explanation for the nearly constant scale height of stellar disks as a function of radius (e.g., de Grijs \& Peletier 1997). The scale height depends on the ratio of the square of the perpendicular velocity dispersion to the surface density. If the radial dispersion induced by clump scattering in our model converts in part to a vertical dispersion through initial and subsequent scattering, and the vertical dispersion also takes on an exponential profile of the same type, then the vertical scale height would be more constant than in the case of an isothermal disk.

The formation of the exponential profile occurs steadily over time. The evolution is faster if the total mass in perturbing clumps is larger. Eventually the profile saturates, evolving very slowly thereafter. This evolution is shown in Figure 2, which plots the surface density and rms velocity dispersion versus radius for 4 different times. The inner exponential has been established by 100 time units and hardly evolves after that. The saturation occurs when every stellar scattering outward is approximately matched by a stellar scattering inward. An example of saturation may occur in the self-gravitating N-body simulation by D'Onghia et al. (2013) where a single massive perturber present for a short time in an already established exponential disk did not change the radial profile noticeably.

\subsection{Near-solid body rotation}

In the case of near-solid body rotation, the gravitational acceleration of the fixed halo is taken to be of the form

$$
g_{\mathrm{SB}}(R)=-\frac{G M_{\mathrm{H}}}{H^{2}}\left(\frac{R}{H}\right)^{0.8} .
$$

The dimensionless length and time are defined in the same way as for the nearly-flat rotation case. Scaling to a dwarf irregular galaxy as an example with this type of rotation curve, we take $H=0.5 \mathrm{kpc}$ and $V_{\mathrm{H}}=50 \mathrm{~km} \mathrm{~s}^{-1}$, so that $T=9.8 \mathrm{Myr}, M_{\mathrm{H}}=2.9 \times 10^{8} M_{\odot}$, and the rotation period at $R=H$ is $61 \mathrm{Myr}$.

The right-hand side of Figure 1 shows a simulation with 20187 non-interacting stars in initially circular orbits with zero initial velocity dispersion and no density gradient. The initial disk size is 9 length units. There are 6 perturbing clumps also inside a radius of 9 units that are in fixed circular orbits with a fractional mass of $0.15 M_{\mathrm{H}}$ each. We pick fewer and more massive clumps in the near-solid body case because dwarf galaxies are smaller compared to the size of a typical star complex than are large spiral galaxies. The softening parameter for the clumps is $0.01 \mathrm{H}$. Recall that the total mass of the galaxy is larger than the scale mass $M_{\mathrm{H}}$, which is only that part inside the halo scale length $H$.

To prevent a single clump from dominating a large number of stellar orbits, we make 
each clump disappear after 10 time units (98 Myr) and reappear instantly at some random azimuthal position with the same radius. This hopping time is about the timescale for giant star formation complexes to form and disperse. Random clump appearance is also consistent with a model in which dark minihalos cross the disk at random places and times.

The results shown in the figure correspond to the state of the disk after 50 time units (490 Myr for the dwarf galaxy scaling). As for the case with a flat rotation curve, the disk with a nearly solid body rotation curve also evolves toward an exponential profile in surface density. The velocity dispersion profile is exponential too, although slightly more flat than in the flat rotation curve case.

Both simulations shown in Figure 1 produce exponential disks, but a near-solid body case tends to form a weak bar and the nearly-flat rotation curve case does not. A bar is a quasi-static configuration for aligned elliptical orbits with a wide range of radii in the solid body case. The perturbers tend to kick stars into such elongated orbits and if enough stars respond to the same perturber, then these stars combine to make an elongation that looks like a bar. While this effect might be reasonable for dwarf irregular galaxies, we chose to mitigate it here by randomizing the perturbations in azimuth. We make this choice because we already know that bars can drive a disk toward an exponential profile, and we want to isolate the effects of clump-like perturbations instead. The bars in our simulations would not promote an exponential disk anyway because the stars that make up the bars are not gravitating.

\section{Discussion}

All of our simulations with sufficiently strong clump-like perturbations drive an initially uniform and cold stellar disk to an exponential form in both surface density and velocity dispersion. The timescale for this evolution depends on the strength of the clump forcing, but for a total relative clump mass of several tens of percent, the time for significant evolution is less than a Gyr. Test cases with no clumps confirmed that the initial profile was stable.

Models with a single massive clump (not shown) required a clump mass equal to $\sim 1 \%$ of the halo mass inside 10 radial units to modify the stellar disk in a reasonable time. Such a massive clump also imprints a permanent asymmetry on the disk, forcing the stars into a banana-shaped region centered on the potential center, with a gap around the clump. Like the classical restricted three-body problem, many of those stars follow banana-orbits in the co-rotating frame. Stars originally near this clump scattered to larger radii on high eccentricity orbits and eventually formed an extended disk with a roughly exponential profile 
over a narrow range of radii (a few length units). The other stars in the disk retained their initially flat profile because they were too distant from the clump to experience significant scattering. This result suggests that each clump contributes independently to the exponential disk, and the ensemble of clumps is what makes the final exponential symmetric.

Randomly appearing and disappearing clumps can stir a stellar disk more than corotating clumps because the time-changing potential from the clumps acts like a nonadiabatic heat source for all of the neighboring stars. This is presumably why the near-solid body model makes an exponential quickly even though the local rate of shear is low. The implication is that star formation clumps or transiting dark mini-halos in dwarf Irregular galaxies promote the evolution of their stellar disks into an exponential form. The same may be true in spiral galaxies, but transient spiral arms may dominate the forcing there.

\section{Conclusions}

Stellar scattering off orbiting or transient clumps can produce exponential profiles in two dimensional stellar disks with a fixed halo potential. No other ingredients are necessary to produce the exponential, making this an attractive solution to the problem of its origin, especially for dwarf Irregulars where there are no spiral arms. Other processes that drive disk evolution, not modeled here, should operate at the same time, but the ubiquity of exponentials from scattering alone ensures that an exponential will eventually develop as long as these other processes do not disrupt it.

We are grateful to D. A. Hunter for comments on the manuscript. 


\section{REFERENCES}

Azzollini, R., Trujillo, I., \& Beckman, J.E. 2008, ApJ, 679, L69

Bakos, J., Trujillo, I., \& Pohlen, M. 2008, ApJ, 683, L103

Barker, M. K., Ferguson, A. M. N., Irwin, M. J., Arimoto, N., \& Jablonka, P. 2012, MNRAS, 419,1489

Bland-Hawthorn, J., Vlajić, M., Freeman, K. C., \& Draine, B. T. 2005, ApJ, 629, 239

Bournaud, F., Elmegreen, B.G., \& Elmegreen, D.M. 2007, ApJ, 670, 237

Crampin, D.J., \& Hoyle, F. 1964, ApJ, 140, 99

Dalcanton J. J., Spergel D. N., Summers F. J., 1997, ApJ, 482, 659

Debattista V. P., Mayer L., Carollo C. M., Moore B., Wadsley J., \& Quinn T., 2006, ApJ, 645,209

de Jong, R. S. 1996, A\&A, 313, 45

de Grijs, R., \& Peletier, R.F. 1997, A\&A, 320, L21

D’Onghia, E., Vogelsberger, M., Hernquist, L. 2013, ApJ, 766, 34

Efstathiou G., 2000, MNRAS, 317, 697

Elmegreen, B.G. \& Hunter, D.A. 2006, ApJ, 636, 712

Erwin, P., Pohlen, M., \& Beckman, J. E. 2008, AJ, 135, 20

Fall S.M., \& Efstathiou G. 1980, MNRAS, 193, 189

Fathi K. 2010, ApJ, 722, L120

Fathi K., Gatchell, M., Hatziminaoglou, E., \& Epinat, B. 2012., MNRAS, 423, L112

Ferguson A. M. N., \& Clarke C. J., 2001, MNRAS, 325, 781

Foyle, K., Courteau, S., \& Thacker, R. J. 2008, MNRAS, 386, 1821

Freeman, K. C. 1970, ApJ, 160, 811

Graham, A.W. \& de Blok, W.J.G. 2001, ApJ, 556, 177 
Grossi, M., Hwang, N., Corbelli, E., Giovanardi, C., Okamoto, S., \& Arimoto, N. 2011, A\&A, 533, A91

Gutiérrez, L, Erwin, P., Aladro, R., \& Beckman, J.E. 2011, AJ, 142, 145

Herrmann, K.A., Hunter, D.A., \& Elmegreen, B.G. 2013, AJ, accepted

Hohl, F. 1971, ApJ, 168, 343

Hunter, D.A.., Elmegreen, B.G., Rubin, V.C., Ashburn, A., Wright, T., Jozsa, G.I.G., \& Struve, C. 2013, submitted to AJ

Hunter, D.A. \& Elmegreen, B.G. 2006, ApJS, 162, 49

Hunter, D.A., Elmegreen, B.G., Oh, S.-H., Anderson, E., Nordgren, T.E., Massey, P., Wilsey, N., \& Riabokin, M., 2011, AJ, 142, 121

Lin D. N. C., \& Pringle J. E., 1987, ApJL, 320, L87

Martínez-Serrano, F. J., Serna, A., Doménech-Moral, M., \& Domínguez-Tenreiro, R. 2009, ApJ, L133

McConnachie, A.W., Ferguson, A.M.N., Irwin, M.J., Dubinski, J., Widrow, L.M., Dotter, A., Ibata, R., Lewis, G.F. 2010, ApJ, 723, 1038

Mestel L. 1963, MNRAS, 126, 553

Peñarrubia, J., McConnachie, A., \& Babul, A. 2006, ApJ, 650, L33

Pohlen, M., \& Trujillo, I. 2006, A\&A, 454, 759

Pustilnik, S.A., Kniazev, A.Y., Lipovetsky, V.A., \& Ugryumov, A.V. 2001, A\&A, 373, 24

Radburn-Smith, D.J., Roškar, R., Debattista, V.P., Dalcanton, J.J., Streich, D., de Jong, R.S., Vlajić, M., Holwerda, B.W., Purcell, C.W., Dolphin, A.E., \& Zucker, Daniel B. 2012, ApJ, 753, 138

Robertson, B., Yoshida, N., Springel, V., \& Hernquist, L. 2004, ApJ, 606, 32

Roškar, R., Debattista, V. P., Quinn, T. R., Stinson, G. S., \& Wadsley, J. 2008, ApJ, 684, L79

Saha, A., et al. 2010, AJ, 140, 1719

Sellwood,J.A., \& Binney,J.J. 2002, MNRAS, 336, 785 
Shi,Y., Helou,G., Yan,L., Armus,L., Wu,Y., Papovich,C., Stierwalt,S. 2011, ApJ, 733, 87

van der Kruit, P. C. 2002, in The Dynamics, Structure \& History of Galaxies: A Workshop in Honour of Professor Ken Freeman. ASP Conference Proceedings, Vol. 273. Eds. G.S. Da Costa and Helmut Jerjen. San Francisco: Astronomical Society of the Pacific, p.7

van Zee, L., Haynes,M. P., Salzer, J. J., \& Broeils, A. H. 1997, AJ, 113, 1618

van Zee, L., Westpfahl, D., Haynes M.P. \& Salzer, J.J. 1998, AJ, 115, 1000

Wang, J.-M., Yan, C.-S., Li, Y.-R., et al. 2009, ApJ, 701, L7

Weiner, B.J., Williams, T.B., van Gorkom, J.H., \& Sellwood, J.A. 2001, ApJ, 546, 916

Wilcots, E.M. \& Miller, B.W. 1998, AJ, 116, 2363

Vlajić, M., Bland-Hawthorn, J., \& Freeman, K. C. 2011, ApJ, 732, id 7

Yoshii, Y., Sommer-Larsen, J. 1989, MNRAS, 236, 779

Younger, J.D., Cox, T.J., Seth, A.C., \& Hernquist, L. 2007, ApJ, 670, 269 

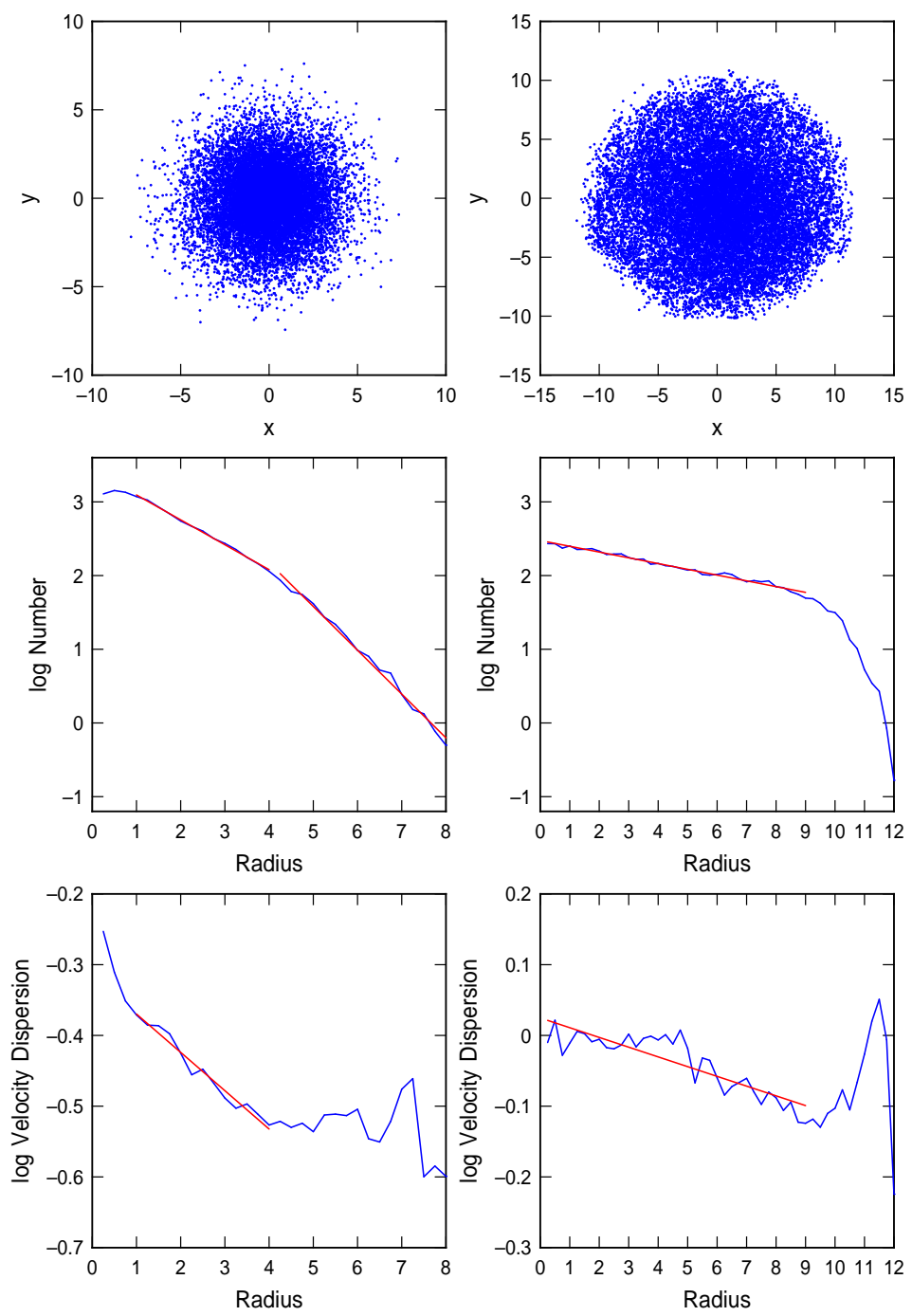

Fig. 1.- (left) The stellar distribution (top), surface density profile (middle) and velocity dispersion profile (bottom) for a non-self gravitating, two-dimensional model stellar disk with a nearly-flat rotation curve imposed by a fixed potential. The surface density is initially flat out to $R=4$ length units ( $8 \mathrm{kpc}$ if scaled to a Milky Way-size galaxy) and then evolves to an exponential by the time shown, which is 300 time units $(\sim 2.7 \mathrm{Gyr})$. The velocity dispersion starts at zero and also evolves to an exponential. This evolution is driven by 70 perturbing clumps in circular orbits with a relative mass of $0.2 \%$ each. The fitted exponentials (red lines) have scale lengths of 3.0 and 1.7 length units for the inner and outer parts of the surface density, and 18.5 length units for the inner part of the velocity dispersion. (right) The same quantities as on the left but now for a near-solid body rotation curve. The initially flat and cold disk extends out to $R=9$ length units $(4.5 \mathrm{kpc}$ for typical scaling of a dwarf Irregular galaxy). The distributions shown are for 50 time units $(\sim 0.49$ Gyr $)$. In this case, the evolution is driven by 6 perturbing clumps in circular orbits with a relative mass of $15 \%$ each; the clumps jump randomly in azimuth every 10 time units (98 Myr) to minimize the formation of a bar. The fitted exponentials have scale lengths of 12.8 units for surface density and 73 units for velocity dispersion. 

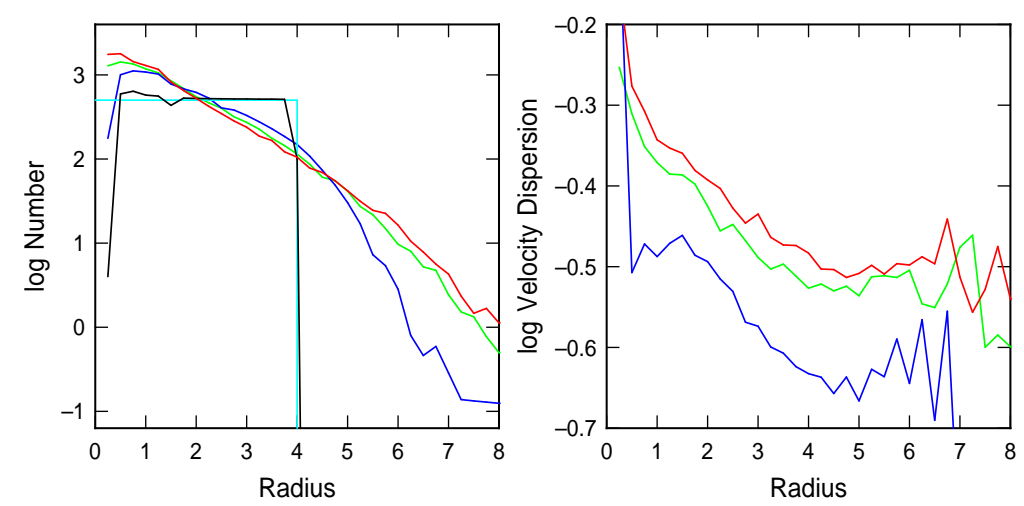

Fig. 2.- (left) Surface density profiles for the nearly-flat rotation curve case at times of 0 (cyan curve), 100 (blue), 300 (green), and 500 (red) time units (0, 890, 2700, and $4450 \mathrm{Myr}$ for the Milky Way scaling). The time 300 case is the same as in figure 1 . The black curve is for the same initial conditions but without perturbers, shown after 100 time units. (right) The profiles of rms velocity dispersion at 100, 300, and 500 time units. 